\title{
Persistence and adherence to ocrelizumab compared with other disease-modifying therapies for multiple sclerosis in U.S. commercial claims data
}

\author{
Natalie J Engmann, PhD, MSc; Danny Sheinson, PhD, MA; Komal Bawa, PharmD; \\ Carmen D Ng, PhD, MA; and Gabriel Pardo, MD
}

\section{What is already known about this subject}

- Medications can only be effective at improving the health of patients when they are used as prescribed, including adherence to the prescribed dosing regimen and persistent use over time.

- A number of disease-modifying therapies (DMTs) for the treatment of multiple sclerosis are available, each with diverse mechanisms of action, modes of administration, and dosing schedules, offering patients the ability to make highly individualized treatment choices that best suit their specific disease characteristics and lifestyle needs.

- Adherence rates ranging from $41 \%$ to $88 \%$ and persistence rates ranging from $50 \%$ to $74 \%$ have been reported for currently available DMTs.

\section{What this study adds \\ - This study provides important evidence on real-world ocrelizumab (OCR) use, demonstrating superior persistence and adherence with OCR vs. other DMTs administered by intravenous (IV), oral, or injectable routes. \\ - Findings from this study support existing evidence demonstrating that patients treated with IV DMTs are more likely to be adherent and persistent to therapy than patients who receive oral or injectable DMTs and, moreover, suggest that reduced frequency of infusions with OCR may further enhance adherence and persistence.}

\section{ABSTRACT \\ BACKGROUND: Ocrelizumab (OCR) is the only disease-modifying therapy (DMT) for both relapsing and primary progressive forms of multiple sclerosis (MS). OCR is given by intravenous (IV) infusion twice a year, which may improve adherence to the dosing sched- ule relative to other MS DMTs that require more frequent administration. Real-world evidence on the persistence and adherence of patients with MS to OCR compared with other DMTs is limited.}

OBJECTIVE: To examine the persistence and adherence to OCR compared with other DMTs for MS in the United States.

METHODS: This analysis was conducted in the PharMetrics Plus commercial claims database and included patients with MS who initiated a new DMT between April 2017 and September 2018. Patients were required to have health plan enrollment for $\geq 1$ year before and after DMT initiation (a subgroup analysis was performed for those with $\geq 18$ months' continuous enrollment after DMT

\section{Author affiliations \\ Natalie J Engmann, PhD, MSc; Danny Sheinson, PhD, MA; Komal Bawa, PharmD; and Carmen D Ng, PhD, MA, Genentech, San Francisco, CA. Gabriel Pardo, MD, Oklahoma Medical Research Foundation, Oklahoma City.}

\section{AUTHOR CORRESPONDENCE:}

Natalie J Engmann, 650.296.2885;

engmann.natalie@gene.com

J Manag Care Spec Pharm 2021;27(5):639-49

Copyright $\odot 2021$, Academy of Managed Care Pharmacy. All rights reserved.

initiation). Persistence was defined as not switching to another DMT and having no gap in coverage of the initiated DMT for $\geq 60$ days during the postinitiation period. The proportion of days covered (PDC) was calculated as the total days covered by the DMT during the postinitiation period divided by the length of the time period (12 or 18 months); $P D C \geq 0.8$ was considered adherent. Multivariable Poisson regression models compared discontinuation (nonpersistence) and nonadherence between OCR users and users of other DMTs grouped by administration route. 
RESULTS: A total of 4,587 patients (OCR, 1,319; injectable, 1,051; oral, 1,876; other IV, 341) were included. The OCR group had the lowest proportion of patients discontinuing at 12 months ( $8 \%$ vs. $28 \%, 32 \%$, and $43 \%$ for other IV, oral, and injectable, respectively) and the highest mean PDC ( $93 \%$ vs. $76 \%, 74 \%$, and $69 \%$, respectively). Compared with patients initiating OCR, adjusted relative risks (RR) of 12-month discontinuation were $3.3(95 \% \mathrm{Cl}=2.3-4.6), 3.8(95 \% \mathrm{Cl}=3.0-4.9)$, and $5.5(95 \% \mathrm{Cl}=4.1-7.5)$ for patients initiating other IV, oral, and injectable DMTs, respectively. Similarly, patients initiating other IV, oral, and injectable DMTs had RRs of $4.9(95 \% \mathrm{Cl}=3.6-6.8)$, $5.1(95 \% \mathrm{Cl}=3.9$ 6.6), and $6.8(95 \% \mathrm{Cl}=5.0-9.3)$ for 12 -month nonadherence compared with OCR. A subgroup of 2,913 patients with 18 months of continuous enrollment had similar trends, with $17 \%$ in the OCR group discontinuing compared with $40 \%, 41 \%$, and $55 \%$ in the other IV, oral, and injectable groups, respectively. Trends over 18 months were consistent with the 12-month analysis in adjusted models.

CONCLUSIONS: Patients initiating OCR had superior persistence and adherence at 12 and 18 months of follow-up compared with patients initiating other MS DMTs. Long-term persistence and adherence should be monitored as OCR experience accrues in a real-world setting.

Multiple sclerosis (MS) is a chronic, progressive, degenerative neurological disease that causes axonal damage and demyelination, resulting in acute and chronic neurological deficits. ${ }^{1} \mathrm{MS}$ is characterized by acute relapse episodes that present as new or worsening neurological symptoms and underlying insidious progression that occurs across functional domains. MS is estimated to affect $>700,000$ people in the United States and is often diagnosed between the ages of 20 and 40 years..$^{2,3}$ Due to the young age of disease onset, the social and economic costs of MS are substantial. ${ }^{4}$

Although there is no cure for MS, guidelines recommend aggressive, efficacious treatment with disease-modifying therapies (DMTs) early in the disease course to mitigate disease progression. ${ }^{5}$ Many DMTs are available for the treatment of relapsing-remitting MS, which constitutes about $85 \%$ of initial MS diagnoses. ${ }^{6}$ More than 15 approved therapies with various mechanisms of action, routes of administration, and dosing schedules are available; thus, the decision of which agent to initiate is highly individualized..$^{-12}$

Medication can maintain or improve health outcomes only when used as prescribed. It is well established that medication persistence and adherence, particularly in chronic diseases, are paramount in the success of treatment. ${ }^{13-16}$ Medication persistence represents continuation on the treatment for the prescribed length of time, while medication adherence is defined as adherence to a medication regimen prescribed by a health care professional with respect to dosage, frequency, and timing. ${ }^{17}$

The abundance of available treatments and personalization of care with regard to patient preference and values theoretically should enhance patients' adherence to MS therapy. However, existing MS DMTs have shown poor to good adherence, with a literature review of 24 studies finding weighted mean adherence to injectable MS DMTs ranging between $41 \%$ and $88 \%{ }^{18}$ The weighted mean adherence across studies included in the review was greater with intramuscular interferon beta-1a (IFN $\beta-1 a)$ administered once weekly (69.4\%) and subcutaneous (SC) IFN $\beta-1 b$ administered every other day (63.8\%) than with SC IFN $\beta-1$ a administered 3 times a week (58.4\%) and glatiramer acetate administered once daily (56.8\%). More recent studies suggest that oral DMTs on average have improved adherence over first-line (SC) therapies, with 1 study suggesting that adherence was between $53 \%$ and $75 \%$ and persistence was $50 \%-74 \%$ over a 1 -year time period. ${ }^{19}$

Nonpersistence and nonadherence to MS DMTs can occur for a variety of reasons and have been shown to lead to poorer outcomes such as increased likelihood of relapse and progression as well as increased health care resource utilization. ${ }^{19,20}$ Reasons for nonpersistence and nonadherence could be lack of efficacy of treatment, safety concerns, and limited access to therapy. Among a large cohort of commercially insured patients with MS initiating DMTs, Burks et al. (2017) observed that patients adherent to DMTs (proportion of days covered $[\mathrm{PDC}] \geq 80 \%$ ) had a significantly decreased likelihood of relapse, emergency department visits, and hospitalization compared with patients who were nonadherent. ${ }^{20}$ Similarly, another analysis of patients with MS from commercial claims data found an association between an increase in PDC and a decrease in the likelihood of inpatient or emergency care visits and fewer days of work loss over follow-up periods of 6-36 months. ${ }^{21}$

Ocrevus (ocrelizumab [OCR]) was approved by the U.S. Food and Drug Administration (FDA) in March 2017 and is the first approved therapy for both relapsing and primary progressive forms of MS. ${ }^{11}$ OCR is administered by intravenous (IV) infusion twice a year (the first of which-called the loading dose-is split between 2 IV infusions approximately 14 days apart), which may improve patients' ability to adhere to the prescribed dosing schedule relative to other MS DMTs that require more frequent administration. However, real-world evidence on the persistence and adherence of patients with MS to OCR compared with other DMTs is limited. The objective of this study was to evaluate the persistence and adherence of patients with MS to OCR compared with other marketed DMTs. 


\section{Methods}

\section{DATA SOURCE}

This analysis was conducted using the PharMetrics Plus commercial claims database. The PharMetrics Plus database is the largest nonpayer-owned integrated claims database of U.S. commercial insurers. The deidentified integrated data include all paid medical and pharmacy (retail and mail order) claims for $>130$ million members from $>70$ U.S. health plans. The integrated database includes inpatient and outpatient claims, diagnoses, and procedures based on International Classification of Diseases, Tenth Revision, Clinical Modification (ICD-10-CM), Healthcare Common Procedure Coding System (HCPCS), and Current Procedural Terminology (CPT) codes.

The secondary data used in this analysis did not contain patient identifiers; therefore, institutional review board approval and patient consent were not applicable.

\section{PATIENT SELECTION/IDENTIFICATION}

Patients who initiated an FDA-approved MS DMT, including OCR, fingolimod, dimethyl fumarate, glatiramer acetate, IFN $\beta-1 \mathrm{a} / \mathrm{b}$, natalizumab, and teriflunomide, between April 1, 2017, and September 30, 2018, were identified. The start of the patient selection period (April 1, 2017) corresponded with the regulatory approval for OCR in the United States (March 28, 2017). The first new DMT prescribed during this period (i.e., the first use of a DMT that had not been prescribed in the previous year) was considered the index DMT, and the date of the first prescription fill or administration of the index DMT was defined as the index date. Note that patients were not required to be DMT naive during the year before the index date; they could have received any DMT that was not the index DMT during that time.

Patients were aged between 18 and 65 years, were commercially or self-insured, and had a minimum of 365 days of continuous enrollment in the health plan before the index date and following the index date (Figure 1). Patients were required to have at least 1 diagnosis of MS (ICD-10-CM: G35) within the continuous enrollment period. Patients initiating alemtuzumab and mitoxantrone were excluded due to insufficient sample size, and, in the case of alemtuzumab, because of its use as an induction therapy. Therapies used off label for MS were also excluded. Patients initiating OCR after the assignment of the permanent J code (J2350, January 2018) or temporary C code (C9494, October 2017) were identified by those codes. To identify OCR use after approval but before the assignment of permanent $\mathrm{J}$ or temporary $\mathrm{C}$ codes, a previously published algorithm was used. ${ }^{22}$ The algorithm combined miscellaneous HCPCS codes J3490, J3590, J9999, or C9399 within 1 day of CPT codes 96413, 96415, 96365, or 96366 to indicate IV infusion procedures, which could potentially identify patients initiating OCR. Patients were required to have (1) either an MS diagnosis on the same day as a miscellaneous J code or use of an approved MS DMT in the year before the first miscellaneous J code and (2) evidence of 2 infusions occurring within 13-21 days, consistent with the OCR first-dose infusion schedule (two $300 \mathrm{mg}$ IV administrations approximately 14 days apart).

For all DMTs, at least 2 prescriptions/administrations of the index DMT were required. For OCR, evidence of both the first and second infusions of the first dose satisfied this requirement.

\section{STUDY MEASURES}

Patient demographics were collected at the index date. DMT treatment as well as clinical and disease characteristics were based on medical and pharmacy claims in the year before the index date. Covariates included age, sex, region, plan type, Charlson Comorbidity Index (CCI), any relapse in the pre-index period, any DMT use in the pre-index period, and MS symptoms characterized by ICD-10-CM codes. MS symptoms included in the analysis have been used in previous claims studies of $\mathrm{MS}^{23}$ and include other causes of myelitis (ICD-10-CM: G04.89); demyelinating disease of the central nervous system (ICD-10-CM: G36.9, G37.9); disorders of the optic nerve and visual pathways (ICD-10-CM: H46); neurogenic bladder (ICD-10-CM: N31.9); other disorders of soft tissues including neuralgia, neuritis, radiculitis, and unspecified (ICD-10-CM: M79.2, M54.10, M54.18); general symptoms such as dizziness and giddiness (ICD-10-CM: R42); and fatigue and malaise (ICD-10-CM: R53.0, R53.1, R53.81-R53.83, G93.3).

Non-OCR MS DMTs were categorized by their route of administration. Self-injectables were defined as DMTs that were administered either by the SC or intramuscular route, including IFN $\beta-1 \mathrm{a} / \mathrm{b}$ and glatiramer acetate. Oral DMTs included dimethyl fumarate, fingolimod, and teriflunomide. IV-infused DMTs included natalizumab and OCR, although they were assessed separately in the analysis (other IV and OCR). Days covered by medication (referred to as coverage) for injectable and orally administered drugs was obtained from the days of supply on drug claims; for other IV and OCR, we used the retreatment interval stated on the prescribing information to determine if a patient was covered by the DMT (28 days for natalizumab and 26 weeks for OCR).

Persistence was defined as having no evidence of switching to another DMT and no gap in coverage of the index DMT of $\geq 60$ days during the post-index year. ${ }^{24}$ Discontinuation was defined as the complement of persistence: evidence of switching to another DMT or a gap in coverage of the 


\section{FIGURE 1 Patient Selection Flowchart}

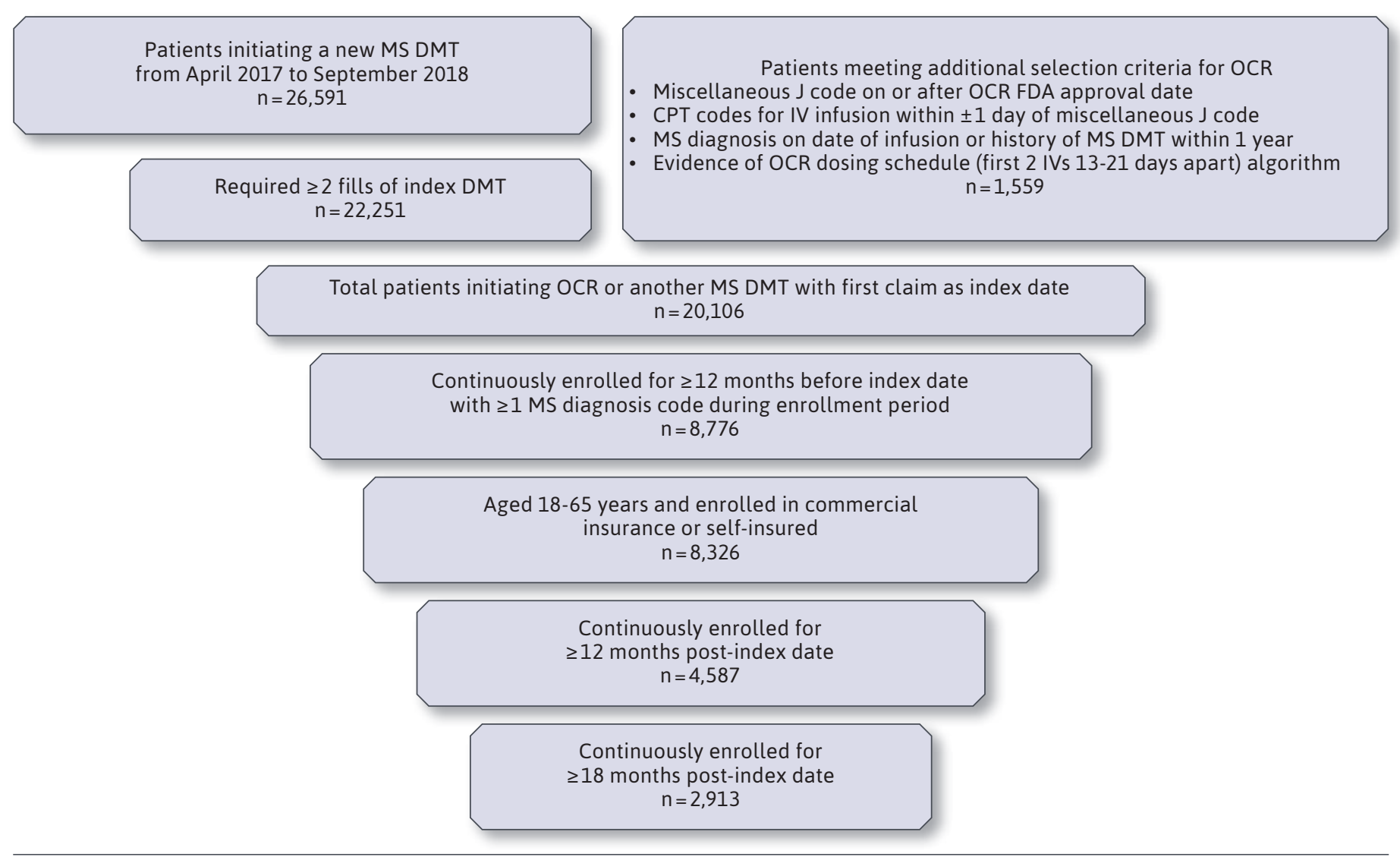

$C P T=$ Current Procedural Terminology; DMT = disease-modifying therapy; FDA=U.S. Food and Drug Administration; IV=intravenous; $M S=$ multiple sclerosis; OCR =ocrelizumab.

index DMT of $\geq 60$ days at any time during the post-index year (overall population) or 18 months post-index (18-month subgroup). Leftover coverage days at a subsequent prescription fill or administration of a DMT were not carried over when assessing the length of the gap in coverage. For OCR, which has a twice-per-year dosing schedule, patients were categorized as nonpersistent if they did not return for their second dose $<60$ days after the end of their first dosing interval (26 weeks).

Adherence was calculated using the PDC measure, which assesses the number of days covered by supply of the index DMT out of a fixed length of time. ${ }^{25}$ The PDC is typically calculated over a 1-year time horizon, among patients with 1 year of follow-up after initiating a new DMT. ${ }^{26}$ In this study, the PDC was calculated as the proportion of days covered by the index DMT during 1 year divided by 365 days post-index (overall population) or during 18 months divided by 547.5 days post-index (18-month subgroup). Similar to the definition of discontinuation, leftover coverage days at a subsequent prescription fill or administration of a DMT were not carried over in the numerator of the PDC calculation. For OCR, this definition of PDC reflects whether patients received their second dose late (or not at all), with $100 \%$ reflecting that the second dose was administered on time. The PDC is reported as an average and dichotomized at $80 \%$; patients with a PDC $\geq 80 \%$ were considered adherent to therapy, and patients with a PDC $<80 \%$ were considered nonadherent to therapy. Persistence and adherence were evaluated at 12 months for the entire patient population and at 18 months for the subgroup who had $\geq 18$ months of follow-up in the health plan. 


\section{TABLE 1 Demographic and Clinical Characteristics $(\mathrm{N}=4,587)$}

\begin{tabular}{|c|c|c|c|c|}
\hline & $\begin{array}{c}\text { OCR } \\
(n=1,319)\end{array}$ & $\begin{array}{l}\text { Other IV DMT } \\
\quad(n=341)\end{array}$ & $\begin{array}{l}\text { Oral DMT } \\
(n=1,876)\end{array}$ & $\begin{array}{l}\text { Injectable DMT } \\
(\mathbf{n}=1,051)\end{array}$ \\
\hline Age, mean (SD), years & 47.9 (9.9) & $41.3(11.0)$ & $44.6(10.4)$ & $43.6(11.1)$ \\
\hline \multicolumn{5}{|l|}{ Age category, $n$ (\%) } \\
\hline 18-34 years & $139(10.5)$ & $104(30.5)$ & $341(18.2)$ & $259(24.6)$ \\
\hline $35-44$ years & $314(23.8)$ & $102(29.9)$ & $565(30.1)$ & $279(26.6)$ \\
\hline $45-54$ years & $475(36.0)$ & $84(24.6)$ & $593(31.6)$ & $303(28.8)$ \\
\hline $55+$ years & $391(29.7)$ & $51(15.0)$ & $377(20.1)$ & $210(20.0)$ \\
\hline $\mathrm{CCl}$, mean (SD) & $0.38(0.85)$ & $0.31(0.88)$ & $0.26(0.7)$ & $0.37(0.97)$ \\
\hline Female, n (\%) & $867(65.7)$ & $247(72.4)$ & $1,427(76.1)$ & $810(77.1)$ \\
\hline \multicolumn{5}{|l|}{ Region, $\mathrm{n}(\%)$} \\
\hline West & $149(11.3)$ & $29(8.5)$ & $204(10.9)$ & $93(8.9)$ \\
\hline East & $266(20.2)$ & 65 (19.1) & $391(20.8)$ & $199(18.9)$ \\
\hline Midwest & $463(35.1)$ & $92(27.0)$ & $617(32.9)$ & $344(32.7)$ \\
\hline South & $441(33.43)$ & $155(45.5)$ & $664(35.4)$ & 415 (39.5) \\
\hline \multicolumn{5}{|l|}{ Plan type, n (\%) } \\
\hline PPO & $1,085(82.3)$ & $292(85.6)$ & $1,583(84.4)$ & $896(85.3)$ \\
\hline HMO & $118(9.0)$ & $28(8.2)$ & $164(8.7)$ & $90(8.6)$ \\
\hline POS & $96(7.3)$ & $18(5.3)$ & $96(5.1)$ & $48(4.6)$ \\
\hline Indemnity/traditional & $19(1.4)$ & $3(0.9)$ & $31(1.7)$ & $12(1.1)$ \\
\hline Consumer-directed health care & $1(0.1)$ & 0 & $1(0.1)$ & $5(0.5)$ \\
\hline Unknown/missing & 0 & 0 & $1(0.1)$ & 0 \\
\hline Pre-index relapse, $\mathrm{n}(\%)$ & $532(40.3)$ & $177(51.9)$ & $574(30.6)$ & $354(33.7)$ \\
\hline Pre-index DMT use, $\mathrm{n}(\%)$ & $876(66.4)$ & $142(41.6)$ & $907(48.4)$ & $191(18.2)$ \\
\hline \multicolumn{5}{|l|}{ Symptom, n (\%) } \\
\hline Other causes of myelitis & $5(0.4)$ & 0 & $6(0.3)$ & $6(0.6)$ \\
\hline Demyelinating diseases of the central nervous system & $90(6.8)$ & $55(16.1)$ & $243(13.0)$ & $251(23.9)$ \\
\hline Disorders of the optic nerve and visual pathways & $124(9.4)$ & $33(9.7)$ & $221(11.8)$ & $151(14.4)$ \\
\hline Neurogenic bladder NOS & $169(12.8)$ & $19(5.6)$ & $69(3.7)$ & $34(3.2)$ \\
\hline $\begin{array}{l}\text { Other disorders of soft tissues: neuralgia, neuritis, } \\
\text { and radiculitis, unspecified }\end{array}$ & $53(4.0)$ & $17(5.0)$ & $54(2.9)$ & $37(3.5)$ \\
\hline General symptoms: dizziness and giddiness & $109(8.2)$ & $34(10.0)$ & $177(9.4)$ & $152(14.5)$ \\
\hline General symptoms: fatigue and malaise & $441(33.4)$ & $105(30.8)$ & $506(27.0)$ & $292(27.8)$ \\
\hline
\end{tabular}

$\mathrm{CCl}=$ Charlson Comorbidity Index; $\mathrm{DMT}=$ disease-modifying therapy; $\mathrm{HMO}=$ health maintenance organization; $I V=$ intravenous; NOS=not otherwise specified; $O C R=$ ocrelizumab; $P O S=$ point of service; $P P O=$ preferred provider organization; $S D=$ standard deviation.

\section{STATISTICAL ANALYSIS}

Patient demographic and disease characteristics were stratified by treatment group (OCR, injectable, oral, and other IV) and summarized by mean and SD or frequency and percentage. The proportion persistent, crude PDC (mean) and proportion adherent ( $\mathrm{PDC} \geq 80 \%$ ) at 12 months (overall population) and 18 months (subgroup with 18-month follow-up) were reported by treatment group. Poisson regression models, which included as covariates individual DMT, age, sex, region, plan type, CCI, relapse in pre-index period, DMT use in pre-index period, and MS symptoms, were used to model the risk of discontinuation and risk of nonadherence (PDC $<80 \%)$ at 12 and 18 months. The relative risk (RR) for OCR compared with each of injectable, oral, and other 


\section{FIGURE 2 Breakdown of DMT Types Initiated by Patients in the Study}

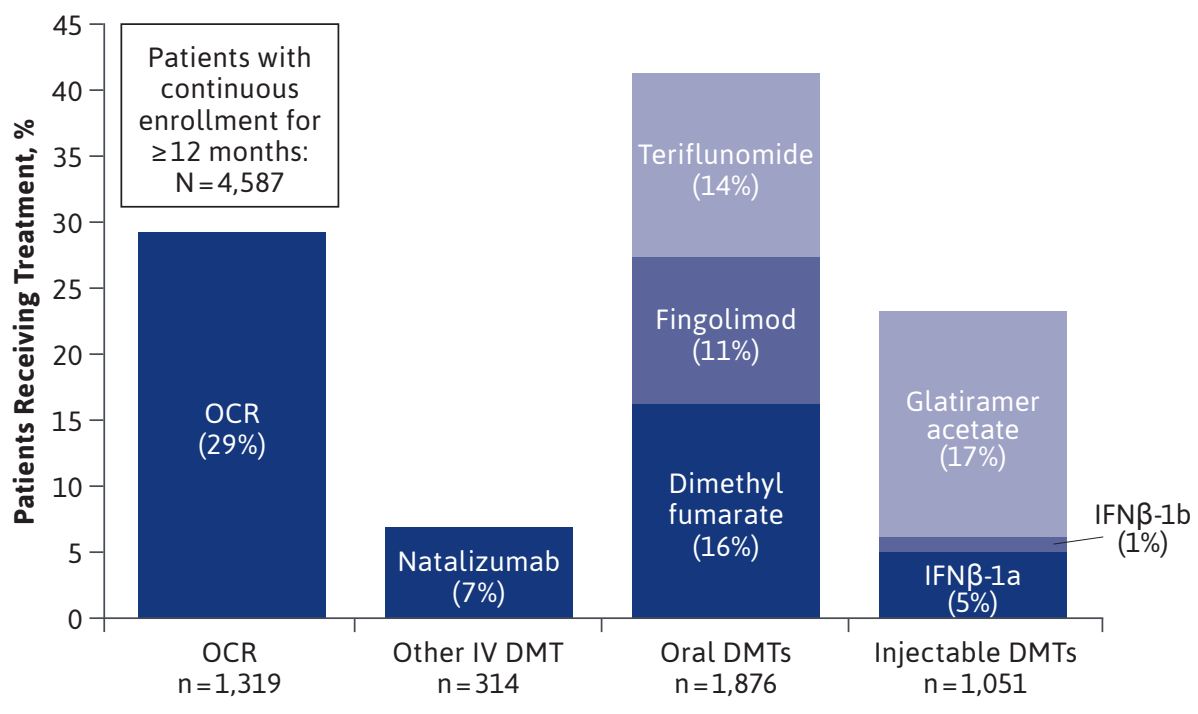

$D M T=$ disease-modifying therapy; IFN $\beta=$ interferon beta; IV =intravenous; $O C R=$ ocrelizumab
In the first year of initiation, $91.6 \%$ of patients initiating OCR were persistent with therapy compared with patients initiating other IV (71.9\%), oral $(67.7 \%)$, and injectable $(56.6 \%)$ therapies. OCR had the lowest proportion of patients discontinuing therapy within 1 year $(8.4 \%$ vs. $28.1 \%$ with other IV, $32.3 \%$ with oral, and $43.4 \%$ with injectable DMTs). Patients discontinuing across OCR and all groups of DMTs were most often classified as discontinued due to gaps in treatment of $\geq 60$ days, as opposed to switching to other DMTs before a gap in therapy. of note, patients classified as having a gap included patients who returned for treatment after $\geq 60$ days as well as those who never returned during the 1-year follow-up.

The proportion of patients switching DMTs (before a gap in treatment) was $0.7 \%$ with OCR and $8.5 \%$ with other IV, $10.5 \%$ with oral, and $16.9 \%$ with injectable DMTs. In adjusted analyses of the risk of discontinuation within 1 year, initiation of other groups of DMTs was associated with greater RR (95\% CI) compared with OCR (other IV DMT, RR=3.3 [2.3-4.6]; oral DMT, $\mathrm{RR}=3.8$ [3.0-4.9]; injectable DMT, $R R=5.5$ [4.1-7.5]; all $\mathrm{P}<0.0001$; Figure 3).

Patients initiating OCR had the highest unadjusted mean PDC over the first year of therapy (93.4\%) compared with the other groups (other IV DMT, 76.1\%; oral DMT, 73.7\%; injectable DMT, 68.7\%; Supplementary Table 1, available in online article). Similarly, patients initiating other IV, oral, and injectable DMTs were more likely to be nonadherent over the year $(\mathrm{PDC}<0.80)$ than patients initiating OCR (Figure 4). In adjusted models, patients initiating other groups of DMTs had greater RR $(95 \% \mathrm{CI})$ of nonadherence over the first year of use compared with OCR (other IV DMT, RR=4.9 [3.6-6.8]; oral DMT, RR =5.1 [3.9-6.6]; injectable DMT, $\mathrm{RR}=6.8$ [5.0-9.3]; all $\mathrm{P}<0.0001$ ). 


\section{FIGURE 3 Persistence to OCR, Other IV, Injectable, and Oral DMTs Over 12 and 18 Months}

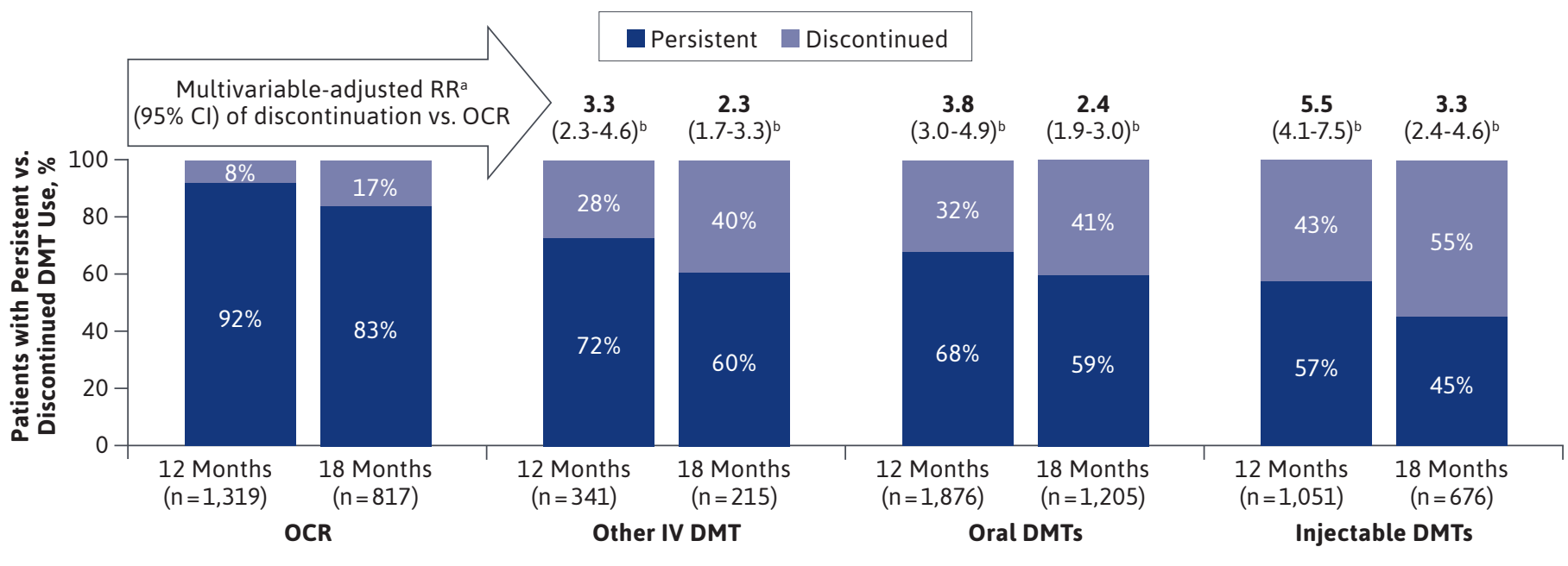

${ }^{a} R R$ was estimated using a multivariable Poisson regression model adjusted for age, sex, payer type (commercial vs. self-insured), insurance plan type, region, relapse in the previous year (yes/no), CCI, DMT use in the pre-index year (yes/no), and presence of MS symptoms (yes/no).

${ }^{b} P<0.0001$.

$\mathrm{CCl}=$ Charlson Comorbidity Index; $\mathrm{Cl}=$ confidence interval; $\mathrm{DMT}=$ disease-modifying therapy; $I \mathrm{~V}=$ intravenous; $\mathrm{MS}=$ multiple sclerosis; $\mathrm{OCR}=\mathrm{ocrelizumab;}$ $R R=$ relative risk.

There were 2,913 patients (64\% of overall study population) who were continuously enrolled in a health plan for at least 18 months after initiating their index DMT. The breakdown of patients initiating OCR $(\mathrm{n}=817 ; 28 \%)$, other IV DMT $(\mathrm{n}=215 ; 7 \%)$, oral DMT $(\mathrm{n}=1,205 ; 41 \%)$, and injectable DMT $(\mathrm{n}=676 ; 23 \%)$ and baseline characteristics by DMT group initiated were similar to the overall cohort. The proportion of patients persistent with therapy at 18 months was $83 \%$ with OCR and $60 \%$ with other IV, $59 \%$ with oral, and $45 \%$ with injectable DMTs. Similar to the 1-year data, a majority of patients were classified as discontinued due to treatment gaps of $\geq 60$ days; $1.4 \%$ of OCR, $12 \%$ of other IV, $12 \%$ of oral, and $22 \%$ of injectable initiators switched to another DMT (before a gap in treatment). In adjusted models, the RR of discontinuation $(95 \% \mathrm{CI})$ with other IV $(\mathrm{RR}=2.3$ [1.7-3.3]), oral $(\mathrm{RR}=2.4[1.9-3.0])$, and injectable $(\mathrm{RR}=3.3[2.4-4.6])$ DMTs (all $\mathrm{P}<0.0001)$ was elevated compared with patients initiating OCR (Figure 3).

The mean PDC over 18 months was 92\% with OCR and $70 \%$ with other IV, $69 \%$ with oral, and $61 \%$ with injectable DMTs. The proportion of patients adherent to therapy (defined as $\mathrm{PDC} \geq 80 \%$ ) was highest with OCR $(86 \%)$, followed by oral (59\%), other IV (57\%), and injectable (45\%)
DMTs. Adjusted results suggest that patients initiating other IV, oral, and injectable DMTs had higher RR (95\% CI) of nonadherence over 18 months compared with OCR (other IV DMT, RR=3.0 [2.2-4.3]; oral DMT, RR=2.9 [2.3-3.8]; injectable DMT, RR=4.3 [3.1-5.9]; all $\mathrm{P}<0.0001$; Figure 4).

\section{SENSITIVITY ANALYSES}

When using a less stringent criterion for persistence that allowed for patients to be persistent despite treatment gaps of $\geq 60$ days throughout the year if they had evidence of DMT coverage at the end of the year, the percentage of patients persistent over 12 months increased slightly for all groups of DMTs (OCR, 94\%; other IV DMT, 76\%; oral DMT, 72\%; injectable DMT, 61\%). The adjusted RR (95\% CI) of discontinuation compared with OCR increased slightly from the more restrictive persistence definition (other IV $\mathrm{DMT}, \mathrm{RR}=4.2$ [2.8-6.2]; oral DMT, $\mathrm{RR}=4.9$ [3.6-6.7]; injectable DMT, RR=7.5 [5.3-10.7]; all $\mathrm{P}<0.0001$; Supplementary Figure 1, available in online article).

After restricting the OCR population to patients identified by permanent J or temporary C codes only ( $\mathrm{n}=574 ; 44 \%)$, 12-month persistence and adherence were similar compared with the full sample, and conclusions remained unchanged (Supplementary Figure 2, available in online article). 


\section{FIGURE 4 Adherence to OCR, Other IV, Injectable, and Oral DMTs Over 12 and 18 Months}

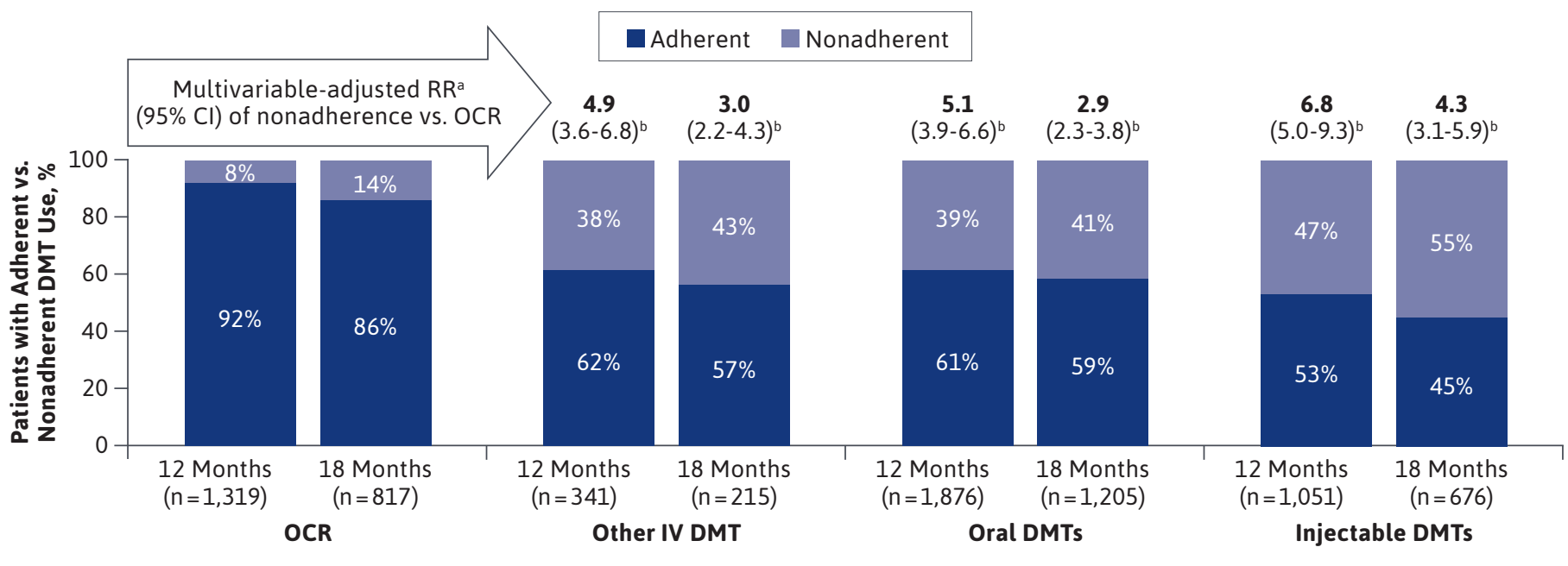

${ }^{a} R R$ was estimated using a multivariable Poisson regression model adjusted for age, sex, payer type (commercial vs. self-insured), insurance plan type, region, relapse in the previous year (yes/no), CCI, DMT use in the pre-index year (yes/no), and presence of MS symptoms (yes/no).

${ }^{b} P<0.0001$.

$\mathrm{CCl}=$ Charlson Comorbidity Index; $\mathrm{Cl}=$ confidence interval; $\mathrm{DMT}=$ disease-modifying therapy; $I \mathrm{~V}=$ intravenous; $\mathrm{MS}=$ multiple sclerosis; $\mathrm{OCR}=\mathrm{ocrelizumab;}$ $R R=$ relative risk.

\section{Discussion}

Our results suggest that patients initiating OCR in a realworld setting were more likely to be persistent and adherent to therapy after 12 and 18 months of follow-up than patients initiating other IV, oral, or injectable DMTs. Persistence and adherence were highest among patients initiating OCR, followed by other IV, oral, and injectable DMTs. Across groups, patients who discontinued were more likely to stop treatment for $\geq 60$ days beyond their coverage period rather than switch immediately to another DMT.

To our knowledge, no previous research has examined persistence and adherence to OCR in a broad U.S. commercially insured population. Our study has several strengths, including a new-user design of nearly 4,000 patients with MS and comparisons across all routes of administration represented in the treatment of MS. Our results for other DMTs are generally consistent with previous studies of other MS DMTs in claims populations, ${ }^{19,24,26,28,29}$ although many of these previous publications stratify results by individual DMTs, whereas we have combined DMTs by route of administration. Nevertheless, the averages by group of DMT fall within the ranges for individual injectable and oral DMTs in published studies using comparable measures. Johnson et al. (2017) studied 1,500 patients initiating oral
DMTs between April and June 2013 and found a 12-month PDC of $68 \%, 71 \%$, and $81 \%$ and $12-$ month persistence of $59 \%, 66 \%$, and $79 \%$ for teriflunomide, dimethyl fumarate, and fingolimod, respectively. ${ }^{19}$ Thomas et al. (2016) noted comparable persistence from a claims analysis of 16,218 patients initiating oral, SC, and IV DMTs. ${ }^{30}$ At 1 year, 65\% of patients were persistent to therapy, and $86 \%$ of these patients were considered adherent to therapy (defined as medication possession ratio $[\mathrm{MPR}] \geq 80 \%$ ). Moreover, persistence and adherence with DMTs were associated with a decreased likelihood of emergency department visits and hospital admission.

Patients taking natalizumab (comprising the "other IV" group) in the present study had a discontinuation rate of $28 \%$, which is consistent with a recent report of $29 \%$ from Duquette et al. (2019), ${ }^{29}$ though slightly slower than an earlier study by Bergvall et al. (2014; reporting 39\% discontinuation). ${ }^{24}$ Taken together, the rates of discontinuation in our study and similar claims studies appear higher than comparable studies conducted using electronic medical record or chart review data. For example, Vollmer et al. (2019) found a discontinuation rate of $32 \%$ over 2 years for natalizumab, with less than $20 \%$ probability of discontinuation in the first 12 months. ${ }^{31}$ One potential explanation for this disparity could be the inclusion of patients in claims 
studies who received natalizumab on an extended interval dosing schedule. Extended interval dosing typically uses a retreatment interval of 5-8 weeks, ${ }^{32}$ as it can reduce the risk of developing progressive multifocal leukoencephalopathy. ${ }^{33}$ Patients receiving natalizumab on an extended interval dosing schedule would explain lower adherence in our and other claims studies and may explain lower persistence if patients received natalizumab at intervals $\geq 8$ weeks (60 days).

The consequences of nonpersistence and nonadherence in MS are both clinical and economic. Burks et al. found that among 12,000 patients with MS initiating oral and injectable DMTs, adherence (PDC $\geq 80 \%$ ) over 1 year reduced the likelihood of relapse by $42 \%$, hospitalization by $52 \%$, and emergency department visits by $38 \%$ compared with nonadherence (all, $\mathrm{P}<0.0001){ }^{20}$ Similarly, Tan et al. (2011) found that patients who were adherent to DMTs incurred lower medical costs than those who were nonadherent (adjusted mean: $\$ 3,380$ [95\% CI $=\$ 3,046-\$ 3,750]$ vs. $\$ 4,348$ $[95 \% \mathrm{CI}=\$ 3,828-\$ 4,940] ; \mathrm{P}=0.003)$, despite having similar MS-related medical costs at index date. ${ }^{34}$

Given the clinical and economic effects of nonadherence, medication persistence and adherence are critical elements of successful disease management in MS. Although there are multiple factors that influence adherence to DMTs, 1 study found that patients with MS cited treatment fatigue, depression, forgetfulness, or a perceived lack of efficacy as challenges to adhering to their prescribed therapy. ${ }^{35}$ Another study reported that patients initiating oral or injectable DMTs were more likely to be nonadherent compared with those initiating IV DMTs, citing the main cause as forgetfulness. ${ }^{36}$ These studies support findings in our own analysis and others that patients treated with infused DMTs are more likely to be persistent and adherent to therapy compared with patients treated with injectable and oral DMTs ${ }^{28,37}$ therapies in which the patient has the full burden of responsibility for administration. Furthermore, our study suggests that IV DMTs with less frequent infusions may have improved persistence and adherence compared with IV DMTs with more frequent dosing.

Although our results are broadly consistent with estimates from previous studies of persistence and adherence in claims databases, direct comparisons are often challenging due to the variety of measures used to evaluate persistence and adherence in the real-world setting. For chronic conditions, including MS, the MPR and PDC measures are most commonly used to assess adherence using claims databases. ${ }^{25}$ The MPR and PDC have important distinctions. The most common variation of MPR measures the coverage of DMT out of the number of days a patient was persistent with therapy, thereby producing estimates that are systematically equal to or higher than the PDC. For a DMT with a twice-a-year dosing schedule, the covered and persistent periods are equal for the first 6 months, leading to an MPR of $100 \%$ for all patients even if they discontinue after the first dose; this estimate provides little utility in decision making. We used PDC in our study because it is generally considered a more conservative measure of adherence. ${ }^{38}$ In addition, dichotomies for adherence versus nonadherence differ, although $80 \%$ is often used in studies of MS because it has been demonstrated to stratify patients exhibiting adverse clinical and economic consequences. ${ }^{20,34,39}$

\section{LIMITATIONS}

This study has important limitations, including comparing adherence to DMTs and groups of DMTs with different dosing schedules and pharmacodynamics. Some DMTs for the treatment of MS are longer acting than others; therefore, a gap in therapy is less likely to have detrimental effects on clinical course compared with another DMT with different pharmacodynamics. In addition, although we grouped the DMTs by route of administration, it is possible that persistence and adherence may vary by drug within categories due to nuances in the dosing schedule (as mentioned above for natalizumab), tolerability, or differences in efficacy. Claimsbased data cannot capture true adherence to injectable and oral DMTs but rather can only inform on whether patients filled the medication; therefore, adherence for these DMTs may be overestimated. Furthermore, due to the nature of claims data, we were not able to measure and adjust for clinical characteristics such as MS type, disease severity, or Expanded Disability Status Scale.

\section{Conclusions}

Persistence and adherence are paramount to achieving therapeutic goals in MS. Our study suggests that patients initiating OCR are more likely to persist with therapy and have better adherence over 12 and 18 months compared with patients initiating other groups of DMTs. The improved adherence over other DMTs is likely partially due to the inherent benefits of OCR as a high-efficacy therapy with an infrequent, twice-yearly dosing schedule. Medication adherence can be a challenge for patients with MS and providers treating MS, and the clinical and economic consequences of nonadherence for patients are well documented. Our findings that OCR has higher persistence and adherence than other intravenously infused, oral, or injectable DMTs for MS can be used to inform patients and providers in support of treatment decision making. 


\section{DISCLOSURES}

This study was funded by Genentech (South San Francisco, CA), a member of the Roche Group. Engmann, Sheinson, Bawa, and Ng are employees of Genentech and shareholders of F. Hoffman-La Roche (Basel, Switzerland).

\section{ACKNOWLEDGMENTS}

The authors acknowledge Erru Yang, Laura Julian, and Edward Neuberger for their contributions to this study. Writing and editorial assistance were provided by Health Interactions (U.S.) and funded by Genentech.

\section{REFERENCES}

1. Goldenberg MM. Multiple sclerosis review. P T. 2012;37(3):175-84.

2. Wallin MT, Culpepper WJ, Campbell JD, et al. The prevalence of MS in the United States: a population-based estimate using health claims data. Neurology. 2019;92(10):e1029-40.

3. Koch-Henriksen N, Sorensen PS. The changing demographic pattern of multiple sclerosis epidemiology. Lancet Neurol. 2010;9(5):520-32.

4. Asche CV, Singer ME, Jhaveri M, Chung H, Miller A. All-cause health care utilization and costs associated with newly diagnosed multiple sclerosis in the United States. J Manag Care Pharm. 2010;16(9):703-12. doi: 10.18553/ jmcp.2010.16.9.703

5. Rae-Grant A, Day GS, Marrie RA, et al. Practice guideline recommendations summary: disease-modifying therapies for adults with multiple sclerosis: report of the Guideline Development, Dissemination, and Implementation Subcommittee of the American Academy of Neurology. Neurology. 2018;90(17):777-88.

6. Weinshenker BG. Natural history of multiple sclerosis. Ann Neurol. 1994;36(Suppl 1):S6-S11.

7. Gilenya (fingolimod) capsules, for oral use. Novartis Pharmaceuticals Corporation. December 2019. Accessed January 25, 2021. https://www.novartis.us/ sites/www.novartis.us/files/gilenya.pdf
8. Betaseron (interferon beta-1b) for injection, for subcutaneous use. Bayer HealthCare Pharmaceuticals. October 2020. Accessed January 25, 2021. https:// labeling.bayerhealthcare.com/html/ products/pi/Betaseron_PI.pdf

9. Plegridy (peginterferon beta-1a) injection, for subcutaneous injection. Biogen. March 2020. Accessed January 25, 2021. https://www.plegridy.com/content/dam/ commercial/plegridy/pat/en_us/pdf/ plegridy-prescribing-information.pdf

10. Tysabri (natalizumab) injection, for intravenous use. Biogen. June 2020. Accessed January 25, 2021. https://www. tysabri.com/content/dam/commercial/ tysabri/pat/en us/pdf/tysabri_prescribing_information.pdf

11. Ocrevus (ocrelizumab) injection, for intravenous use. Genentech. December 2020. Accessed January 25, 2021. https:// www.gene.com/download/pdf/ocrevus prescribing.pdf

12. Tecfidera (dimethyl fumarate) delayedrelease capsules, for oral use. Biogen. February 2020. Accessed January 25, 2021. https://www.tecfidera.com/content/ dam/commercial/tecfidera/pat/en_us/ pdf/full-prescribing-info.pdf

13. World Health Organization. Adherence to Long-Term Therapies: Evidence for Action. Geneva, Switzerland: World Health Organization; 2003.

14. Osterberg L, Blaschke T. Adherence to medication. N Engl J Med. 2005;353(5):487-97.

15. Cramer JA, Benedict A, Muszbek N, Keskinaslan A, Khan ZM. The significance of compliance and persistence in the treatment of diabetes, hypertension and dyslipidaemia: a review. Int J Clin Pract. 2008;62(1):76-87.

16. Sokol MC, McGuigan KA, Verbrugge RR, Epstein RS. Impact of medication adherence on hospitalization risk and healthcare cost. Med Care. 2005;43(6):521-30.

17. Cramer JA, Roy A, Burrell A, et al. Medication compliance and persistence: terminology and definitions. Value Health. 2008;11(1):44-47.
18. Menzin J, Caon C, Nichols C, White LA, Friedman M, Pill MW. Narrative review of the literature on adherence to diseasemodifying therapies among patients with multiple sclerosis. J Manag Care Pharm. 2013;19(1 Suppl A):S24-S40. doi: 10.18553/ jmcp.2013.19.s1.S24

19. Johnson KM, Zhou H, Lin F, Ko JJ, Herrera V. Real-world adherence and persistence to oral disease-modifying therapies in multiple sclerosis patients over 1 year. J Manag Care Spec Pharm. 2017;23(8):844-52. doi: 10.18553/ jmcp.2017.23.8.844

20. Burks J, Marshall TS, Ye X. Adherence to disease-modifying therapies and its impact on relapse, health resource utilization, and costs among patients with multiple sclerosis. Clinicoecon Outcomes Res. 2017;9:251-60.

21. Yermakov S, Davis M, Calnan M, et al. Impact of increasing adherence to disease-modifying therapies on healthcare resource utilization and direct medical and indirect work loss costs for patients with multiple sclerosis. J Med Econ. 2015;18(9):711-20.

22. Engmann NJ, Yang E, Julian L, Yeh WS, Whiteley J. Early experience with ocrelizumab: patient characteristics from a large insurance claims database. Poster presented at: 34th Congress of the European Committee for Treatment and Research in Multiple Sclerosis (ECTRIMS); October 10, 2018; Berlin, Germany. [abstract EP1706]. Accessed January 25, 2021. https://onlinelibrary. ectrims-congress.eu/ectrims/2018/ ectrims-2018/229543/natalie.j.engmann. early.experience.with.ocrelizumab.patient. characteristics.html

23. Boster A, Nicholas J, Wu N, et al. Comparative effectiveness research of disease-modifying therapies for the management of multiple sclerosis: analysis of a large health insurance claims database. Neurol Ther. 2017;6(1):91-102. 
24. Bergvall N, Petrilla AA, Karkare SU, et al. Persistence with and adherence to fingolimod compared with other diseasemodifying therapies for the treatment of multiple sclerosis: a retrospective U.S. claims database analysis. J Med Econ. 2014;17(10):696-707.

25. Canfield SL, Zuckerman A, Anguiano RH, et al. Navigating the Wild West of medication adherence reporting in specialty pharmacy. J Manag Care Spec Pharm. 2019;25(10):1073-77. doi: 10.18553/ jmcp.2019.25.10.1073

26. Agashivala N, Wu N, Abouzaid S, et al. Compliance to fingolimod and other disease modifying treatments in multiple sclerosis patients, a retrospective cohort study. BMC Neurol. 2013;13(1):138.

27. Lenth R, Buerkner P, Herve M, Love J, Riebl H, Singmann H. emmeans: estimated marginal means, aka least-squares means. February 3, 2021. Accessed February 8, 2021. https://CRAN.R-project.org/ package $=$ emmeans

28. Halpern R, Agarwal S, Dembek C, Borton L, Lopez-Bresnahan M.

Comparison of adherence and persistence among multiple sclerosis patients treated with disease-modifying therapies: a retrospective administrative claims analysis. Patient Prefer Adherence. 2011;5:73-84.
29. Duquette P, Yeung M, Mouallif S, Nakhaipour HR, Haddad P, Schecter R. A retrospective claims analysis: compliance and discontinuation rates among Canadian patients with multiple sclerosis treated with disease-modifying therapies. PLoS One. 2019;14(1):e0210417.

30. Thomas NP, Curkendall S, Farr AM, Yu E, Hurley D. The impact of persistence with therapy on inpatient admissions and emergency room visits in the US among patients with multiple sclerosis. J Med Econ. 2016;19(5):497-505.

31. Vollmer BL, Nair KV, Sillau S, et al. Natalizumab versus fingolimod and dimethyl fumarate in multiple sclerosis treatment. Ann Clin Transl Neuro. 2019;6(2):252-62.

32. Yamout BI, Sahraian MA, Ayoubi NE, et al. Efficacy and safety of natalizumab extended interval dosing. Mult Scler Relat Disord. 2018;24:113-16.

33. Ryerson LZ, Foley J, Chang I, et al. Risk of natalizumab-associated PML in patients with MS is reduced with extended interval dosing. Neurology. 2019;93(15):e1452-62.

34. Tan H, Cai Q, Agarwal S, Stephenson JJ, Kamat S. Impact of adherence to disease-modifying therapies on clinical and economic outcomes among patients with multiple sclerosis. Adv Ther. 2011;28(1):51-61.
35. Treadaway K, Cutter G, Salter A, et al. Factors that influence adherence with disease-modifying therapy in MS. J Neurol. 2009;256(4):568-76.

36. Morillo Verdugo R, Ramírez Herráiz E, Fernández-Del Olmo R, Roig Bonet $\mathrm{M}$, Valdivia García M. Adherence to diseasemodifying treatments in patients with multiple sclerosis in Spain. Patient Prefer Adherence. 2019;13:261-72.

37. Bonafede MM, Johnson BH, Wenten M, Watson C. Treatment patterns in diseasemodifying therapy for patients with multiple sclerosis in the United States. Clin Ther. 2013;35(10):1501-12.

38. Nau DP. Proportion of days covered (PDC) as a preferred method of measuring medication adherence. Pharmacy Quality Alliance. 2011. Accessed January 25, 2021. http://ep.yimg.com/ty/cdn/epill/pdcmpr.pdf

39. Oleen-Burkey MA, Dor A, Castelli-Haley J, Lage MJ. The relationship between alternative medication possession ratio thresholds and outcomes: evidence from the use of glatiramer acetate. J Med Econ. 2011;14(6):739-47. 